\title{
Percepção e condição de saúde bucal de pessoas com deficiência visual no Município de São José do Rio Preto - SP
}

\author{
Perception and oral health status of visually impaired people in the City of São José do Rio Preto -
}

São Paulo

Percepción y estado de salud bucal de las personas con discapacidad visual en la Ciudad de São

José do Rio Preto - SP

Recebido: 23/06/2021 | Revisado: 01/07/2021 | Aceito: 05/07/2021 | Publicado: 16/07/2021

\author{
Cléa Adas Saliba Garbin \\ ORCID: https://orcid.org/0000-0001-5069-8812 \\ Universidade Estadual Paulista, Brasil \\ E-mail: clea.saliba-garbin@unesp.br \\ Aretuza Marques Bottós \\ ORCID: https://orcid.org/0000-0001-7613-5487 \\ Universidade Estadual Paulista, Brasil \\ E-mail: aretuza.bottos@unesp.br \\ Mariana Martins Ortega \\ ORCID: https://orcid.org/0000-0002-2828-3183 \\ Universidade Estadual Paulista, Brasil \\ E-mail: mazinha_ortega @hotmail.com \\ Artênio José Î́sper Garbin \\ ORCID: https://orcid.org/0000-0002-7017-8942 \\ Universidade Estadual Paulista, Brasil \\ E-mail: artenio.garbin@unesp.br \\ Suzely Adas Saliba Moimaz \\ ORCID: https://orcid.org/0000-0002-4949-529X \\ Universidade Estadual Paulista, Brasil \\ E-mail: suzely.moimaz@unesp.br
}

\begin{abstract}
Resumo
Objetivou-se avaliar as condições e percepções de saúde bucal de pessoas com deficiência visual. Trata-se de um estudo transversal, de caráter exploratório, quantitativo, realizado com 72 portadores de deficiência visual em instituto para cegos do interior do estado de São Paulo. Foram aplicados os questionários OHIP-14 para adultos, CPQ-11-14 para crianças e índice CPOD para avaliar a condição de saúde bucal. Variáveis sociodemográficas foram consideradas. Os dados foram analisados de forma descritiva, através de frequências relativas, absolutas e analíticas. Variáveis categóricas foram comparadas por meio dos testes Qui-quadrado e teste exato de Fisher, significância 5\%. O CPOD médio foi 12,8 para a idade média 34,5 anos, $50 \%$ eram totalmente cegos e $65,3 \%$, do sexo masculino. Grande parte relatou necessidade de ir ao dentista $(66,7 \%)$, apresentando significância estatística associada à percepção de saúde bucal $(\mathrm{p}=0,024)$. Entre os grupos cegueira total e baixa visão, a dimensão desconforto psicológico foi estatisticamente significativa $(\mathrm{p}=0,0008)$, demonstrando maior impacto na qualidade de vida do grupo cegueira total. Conclui-se que os indivíduos com deficiência visual possuem boa percepção de sua saúde bucal e condição bucal compatível com a média populacional brasileira. No entanto, ainda são necessárias ações de promoção da saúde bucal específicas para atender suas carências.
\end{abstract}

Palavras-chave: Pessoas com deficiência visual; Saúde bucal; Epidemiologia; Qualidade de vida.

\begin{abstract}
The objective was to evaluate the oral health conditions and perceptions of people with visual impairment. This is a cross-sectional, exploratory, quantitative study, carried out with 72 visually impaired people in an institute for the blind in the interior of the state of São Paulo. Were applied the OHIP-14 questionnaires for adults, CPQ-11-14 for children and DMFT index to assess oral health condition. Sociodemographic variables were considered. The data were analyzed in a descriptive way, through relative, absolute and analytical frequencies. Categorical variables were compared using the Chi-square test and Fisher's exact test, with a 5\% significance level. The average DMFT was 12.8 for the average age 34.5 years, $50 \%$ were totally blind and $65.3 \%$, male. Most of them reported the need to go to the dentist $(66.7 \%)$, with statistical significance associated with the perception of oral health $(p=0.024)$. Among the total blindness and low vision groups, the psychological discomfort dimension was statistically significant $(p=0.0008)$, demonstrating a greater impact on the quality of life of the total blindness group. It is concluded that individuals with
\end{abstract}


visual impairment have a good perception of their oral health and oral condition compatible with the Brazilian population average. However, specific oral health promotion actions are still needed to meet their needs.

Keywords: Visually impaired persons; Oral health; Epidemiology; Quality of life.

\section{Resumen}

El objetivo fue evaluar las condiciones de salud bucal y las percepciones de las personas con discapacidad visual. Este es un estudio transversal, exploratorio, cuantitativo, realizado con 72 personas con discapacidad visual en un instituto para ciegos del interior del estado de São Paulo. Se aplicaron cuestionarios OHIP-14 para adultos, CPQ-11-14 para niños y el índice CPOD para evaluar el estado de salud bucal. Se consideraron variables sociodemográficas. Los datos se analizaron de forma descriptiva, utilizando frecuencias relativas, absolutas y analíticas. Las variables categóricas se compararon mediante las pruebas de Chi-cuadrado y Exacto de Fisher, con un nivel de significancia del 5\%. El CPOD promedio fue de 12,8 para la edad promedio de 34,5 años, el 50\% eran totalmente ciegos y el 65,3\%, varones. La mayoría refirió necesidad de acudir al dentista $(66,7 \%)$, con significación estadística asociada a la percepción de salud bucal $(\mathrm{p}=0,024)$. Entre los grupos de ceguera total y baja visión, la dimensión de malestar psicológico fue estadísticamente significativa $(\mathrm{p}=0,0008)$, demostrando un mayor impacto en la calidad de vida del grupo de ceguera total. Se concluye que las personas con discapacidad visual tienen una buena percepción de su salud bucal y condición bucal compatible con el promedio de la población brasileña. Sin embargo, aún se necesitan acciones específicas de promoción de la salud bucodental para satisfacer sus necesidades.

Palabras clave: Personas con daño visual; Salud bucal; Epidemiología; Calidad de vida.

\section{Introdução}

A maioria das informações e percepções sobre o desenvolvimento do ser humano surge da observação de outros de sua espécie, principalmente no que tange a sua interação social e diferentes mecanismos e métodos de saúde e higiene corporal. A visão é um dos cinco sentidos principais do corpo humano e pode-se dizer que o dominante entre eles, sendo um dos maiores transmissores de dados ao cérebro (Teixeira, 2006; Magill, 2000). Dessa forma, o impacto da deficiência visual na vida das pessoas é um fator que merece atenção e reflexão.

É conveniente estabelecer a definição do termo "deficiência visual" para este estudo, que no caso em questão refere-se ao espectro da cegueira total à baixa visão, caracterizada pela capacidade funcional alterada devido a fatores relacionados a uma redução significativa da acuidade e uma redução significativa do campo visual e da sensibilidade aos contrastes (Amorim, Medeiros Neta, \& Guimarães, 2020). É importante ressaltar que a visão é o mais importante canal de relacionamento entre o indivíduo e o mundo exterior, uma vez que é responsável por captar os registros próximos ou distantes, permitindo a organização de informações em nível cerebral, trazidas por outros órgãos dos sentidos (Gil, 2000).

Segundo dados do censo realizado em 2010 pelo Instituto Brasileiro de Geografia e Estatística (IBGE), das mais de 6,5 milhões de pessoas com alguma deficiência visual, 8,13\% são incapazes de enxergar (totalmente cegos) e 93,17\% possuem baixa visão ou visão subnormal (grande e permanente dificuldade de enxergar). Outros 29 milhões de indivíduos declararam possuir alguma dificuldade permanente de enxergar, ainda que usando óculos ou lentes (IBGE, 2010; Silva, Marcelino, Gagliardo, Santos, \& Albuquerque, 2020).

O homem é um ser social, habituado a nascer, crescer e morrer em comunidade, cuja criação de laços e o seu próprio reconhecimento como indivíduo surge da observação e convivência com seu grupo (Honneth, 2013). Assim, o sistema sensorial é um elemento fundamental para seu desenvolvimento pleno.

A constatação da deficiência visual ainda é algo que gera inúmeras preocupações no seio familiar e comunitário, pois além dos obstáculos físicos que interferem na locomoção e acessibilidade, inúmeras são as barreiras sociais e culturais presentes em suas vidas, dificultando o alcance aos direitos de comunicação, socialização e integração (Oliveira, Silva, Dias, \& Martins, 2020). A falta de inclusão no mercado de trabalho, a impossibilidade de uma vida independente e segura são exemplos dos enfretamentos vividos por essa população (Fundação Dorina Nowill para Cegos, 2021).

Como tentativa de amenizar os danos, foi criado por meio de decreto 7.612, de 17 de novembro de 2011 o programa "Viver sem Limites - Plano Nacional dos Direitos da Pessoa com Deficiência", elaborado com a participação de mais de 15 
ministérios e do CONADE (Conselho Nacional dos Direitos da Pessoa com Deficiência). O plano tem por objetivo promover programas e ações para este público, por meio da integração e articulação de políticas públicas. Prevê também o exercício pleno e equitativo dos direitos das pessoas com deficiência, sendo os principais eixos de atuação o acesso à educação, saúde, inclusão social e a acessibilidade (Brasil, 2011; Brasil, 2013).

No que se refere a saúde, o Plano Nacional dos Direitos da Pessoa com Deficiência visa reconhecer as necessidades deste público para então incluí-los em toda a rede de serviços do SUS, garantindo o acesso integral à saúde da pessoa com deficiência, da atenção primária à especializada (Brasil, 2010; Brasil, 2017). Entretanto, na prática tem se observado a existência de barreiras que impedem o acesso destes indivíduos à atenção pretendida, seja por falta de acessibilidade ou por falta de pessoal capacitado para receber este público (Lima, Baptista, \& Vargas, 2017).

$\mathrm{Na}$ área odontológica, é de extrema importância que existam protocolos de atendimento e programas de promoção de saúde específicos para pessoas com deficiência, destacando aqui a deficiência visual, uma vez que as maiores necessidades odontológicas desse grupo estão relacionadas às suas dificuldades em aprender e manter uma higiene adequada, distinguindose dos videntes pela menor capacidade de remoção da placa bacteriana e da identificação dos sinais de cárie dentária precoce (Brown, 2008; Batista, Turrini, Moraes \& Rolim, 2003; Rath, Bosco, Almeida \& Moreira, 2001). Dessa forma, indivíduos com deficiência visual são mais suscetíveis ao desenvolvimento de cáries em comparação com videntes (Al Sarheed, Alkhatib, \& Hunt, 2006; Yalcinkaya, \& Atalay, 2006).

Assim, é importante que essa população tenha uma boa percepção de sua condição bucal para que doenças sejam prevenidas ou tratadas quando necessário. Sendo assim, este estudo teve como objetivo avaliar a percepção e a condição de saúde bucal em pessoas com deficiência visual.

\section{Metodologia}

Trata-se de um estudo transversal, de caráter exploratório, quantitativo, realizado no primeiro semestre de 2018, no qual participaram 72 portadores de deficiência visual, participantes de um instituto para cegos do interior do estado de São Paulo.

O instituto foi fundado em 1948 e atende mais de 100 municípios, sendo a única entidade para cegos na região, contando com a participação de 150 indivíduos, no período de realização deste estudo. Oferece atendimento de habilitação, reabilitação e apoio social, psicológico, terapêutico e pedagógico aos deficientes e suas famílias. A unidade prioriza atendimentos a famílias em situação de vulnerabilidade social, com o intuito de promover a inclusão social por meio da capacitação e inserção no mundo do trabalho, desenvolvendo a autonomia e autoestima dos beneficiários.

Para composição da amostra, foram incluídos todos os indivíduos que atendiam aos critérios de inclusão estabelecidos em: capacidade de responder ao questionário, assinatura do termo de consentimento livre e esclarecido e presença nos dias das visitas. Foram excluídos os indivíduos com deficiência intelectual ou que não entendessem as questões, bem como sujeitos que não compareceram nos dias das visitas.

É importante destacar que cálculos foram realizados para estimar a amostra de acordo com a população do Instituto, com nível de confiança de 90\% e erro amostral de 7\%, o que resultou em um número representativo de 72 indivíduos (cegueira total e baixa visão). Observa-se também que a amostra foi composta por adultos e crianças, com idades variando de 07 a 80 anos.

Os instrumentos de coleta utilizados para avaliar a percepção em saúde bucal foram: Oral Health Impact Profile (OHIP-14) (Slade, 1997) para adultos e Child Perceptions Questionnaire (CPQ-11-14) (Jokovic, Locker, Stephens, Kenny, \& Thompson, 2003), já validados em outros trabalhos, dispensando a necessidade de estudo piloto. Variáveis sociodemográficas como sexo, idade, tipo de deficiência visual e o tempo como portador dessa deficiência também foram analisadas. 
Desenvolvido por Slade (1997), em sua forma já traduzida e validada para o português brasileiro (Oliveira, \& Nadanovsky, 2005), o Oral Health Impact Profile (OHIP-14) é um questionário que abrange sete dimensões, cada uma com dois itens, relacionados à limitação funcional, dor física, desconforto psicológico, incapacidade física, incapacidade psicológica, incapacidade social e desvantagem social. A desvantagem social foi excluída dos resultados, uma vez que o questionário utilizado com as crianças não abrange esta dimensão.

O Impact Short Form: 16 (ISF-16) é o pequeno questionário CPQ11-14 desenvolvido no Canadá por Jokovic et.al. (2003), para avaliar a percepção da saúde bucal em crianças, o qual o possui uma versão traduzida e adaptada para aplicação no Brasil (Barbosa \& Gavião, 2011). Os itens tratam de eventos ocorridos nos três meses anteriores. As medidas são estruturalmente compostas por 16 itens, distribuídos em 4 subescalas: sintomas orais, limitações funcionais, bem-estar emocional e bem-estar social.

As opções de resposta dos dois questionários correspondem a uma escala Likert, de forma que cada um pode receber uma série de pontos (nunca $=0$, raramente $=1$, às vezes $=2$, constantemente $=3$ e sempre $=4)$. A soma dos pontos $($ de 0 a 4$)$ atribuídos às respostas dadas a cada item foi realizada de forma a gerar uma pontuação global do OHIP-14, com valores variando de 0 a 56. Além disso, para análise de associações, no OHIP-14 os valores foram dicotomizados na presença de impacto na qualidade de vida (pelo menos uma resposta "às vezes", "constantemente" ou "sempre") e nenhum impacto "nunca" e "raramente" em todos os itens de cada dimensão).

Foram realizadas duas visitas semanais, durante 3 meses, para que os instrumentos fossem aplicados individualmente por um examinador calibrado, que anotava todas as respostas dos participantes. Essa opção foi adotada devido à condição da população estudada e à impossibilidade de transcrição do questionário para Braille, uma vez que nem todos os respondentes dominavam a técnica.

O termo de consentimento livre e esclarecido foi lido em voz alta pela pesquisadora e, ao concordar com a pesquisa, o entrevistado assinava com impressão digital.

Após a coleta de dados, as orientações de higiene bucal foram adaptadas individualmente. Todos os entrevistados receberam as orientações e a escovação supervisionada foi realizada apenas com as crianças, pois os adultos não portavam suas respectivas escovas, com esse grupo foi realizada a limpeza com gaze, em seguida, o exame clínico foi realizado para avaliar doenças orais primárias.

Para o diagnóstico da cárie dentária, foi utilizado o índice CPO-D, que avalia os dentes cariados, perdidos e obturados, conforme recomendação da Organização Mundial da Saúde (WHO, 2013). Ressalta-se que este índice foi aplicado por um pesquisador calibrado por meio de um teste intraexaminador, com índice kappa de 0,89 , em luz natural, com espelho e sonda correspondentes.

Foi realizada análise descritiva dos dados, na forma de frequência relativa, absoluta e analítica. Além disso, as variáveis categóricas foram comparadas por meio do teste Qui-quadrado e teste exato de Fisher, com nível de significância de 5\% ( $\mathrm{p}<0,05$ ). Para análise do índice CPOD foi calculada a mediana da população estudada e, a partir dela, o índice foi classificado em alto e baixo; o índice de idade média também foi calculado. Os dados foram tabulados pelo Epi Info 7.2, e a análise realizada pelo BioEstat 5.3 (Ayres, Ayres Júnior, Ayres, \& Santos, 2007).

O presente estudo foi aprovado pelo Comitê de Ética em Pesquisa da FOA-UNESP, por meio da Plataforma Brasil (CAAE: 76484317.8.0000.5420). É importante destacar também que a pesquisa foi norteada pela Resolução 466/2012 do Conselho Nacional de Saúde (CNS) do Ministério da Saúde - Brasil.

\section{Resultados}

Do total de 72 participantes, $65,3 \%$ eram do sexo masculino, com média de idade de 34,6 anos e $50 \%$ tinham cegueira 
total. Grande parte relatou necessidade de ir ao dentista $(66,7 \%)$. Em relação ao estado dos dentes e gengivas, a maioria, $54,2 \%$, classificou como bom. Além disso, entre os entrevistados, 55,6\% tiveram uma boa percepção sobre sua saúde bucal. O índice CPO-D foi classificado como baixo, em relação à mediana, para a maioria dos pesquisados (Tabela 1).

Tabela 1. Caracterização da amostra de pessoas com deficiência visual, participantes de uma instituição no município de São José do Rio Preto, São Paulo. 2019.

\begin{tabular}{|c|c|c|}
\hline Variáveis & $\mathrm{n}$ & $\%$ \\
\hline \multicolumn{3}{|l|}{ Gênero } \\
\hline Masculino & 47 & 65.3 \\
\hline Feminino & 25 & 34.7 \\
\hline \multicolumn{3}{|l|}{ Faixa etária } \\
\hline Adultos & 47 & 65.3 \\
\hline Crianças & 25 & 34.7 \\
\hline \multicolumn{3}{|l|}{ Tipo de deficiência } \\
\hline Cegueira total & 36 & 50.0 \\
\hline Baixa Visão & 36 & 50.0 \\
\hline \multicolumn{3}{|l|}{$\begin{array}{l}\text { Você acha que precisa ir ao } \\
\text { dentista? }\end{array}$} \\
\hline Não & 24 & 33.3 \\
\hline Sim & 48 & 66.7 \\
\hline $\begin{array}{l}\text { Como estão seus dentes e } \\
\text { gengivas? }\end{array}$ & & \\
\hline Ruins & 10 & 13.9 \\
\hline Regulares & 23 & 31.9 \\
\hline Bons & 39 & 54.2 \\
\hline \multicolumn{3}{|l|}{ Percepção de saúde bucal } \\
\hline Boa & 40 & 55.6 \\
\hline Ruim & 32 & 44.4 \\
\hline \multicolumn{3}{|l|}{ CPOD } \\
\hline Alto & 33 & 45.8 \\
\hline Baixo & 39 & 54.2 \\
\hline Total & 72 & 100.0 \\
\hline
\end{tabular}

Fonte: Autores.

Houve prevalência de dentes obturados na faixa etária de 5 a 18 anos (78,3\%), com CPO-D médio de 3,32. O CPOD médio na faixa etária de 22 a 49 anos foi de 15,3, com maior percentual de dentes obturados (73,7\%). A faixa etária de 50 a 80 anos apresentou CPO-D médio de 21,7, composta em sua maioria por dentes cariados (80,9\%). Em relação ao índice CPO-D total, 49,3\% dos dentes foram obturados, 46,1\% perdidos e 4,6\% cariados, com CPO-D médio de 12,8\% para a média de idade de 34,5 anos (Tabela 2). 
Tabela 2. Distribuição dos números, médias e percentuais dos componentes do índice CPOD em pessoas com deficiência visual por faixa etária, participantes de uma instituição no município de São José do Rio Preto, São Paulo. 2019.

\begin{tabular}{|c|c|c|c|c|c|c|c|c|c|c|c|c|}
\hline \multirow{2}{*}{ Variáveis } & \multicolumn{3}{|c|}{5 a 18 anos $(n=25)$} & \multicolumn{3}{|c|}{22 a 49 anos $(n=28)$} & \multicolumn{3}{|c|}{50 a 80 anos $(n=19)$} & \multicolumn{3}{|c|}{ Total $(n=72)$} \\
\hline & $\mathrm{n}$ & Média & $\%$ & $\mathrm{n}$ & Média & $\%$ & $\mathrm{n}$ & Média & $\%$ & $\mathrm{n}$ & Média & $\%$ \\
\hline Dentes cariados & 18 & 0.72 & 21.69 & 20 & 0.71 & 4.66 & 4 & 0.21 & 0.97 & 42 & 0.58 & 4.56 \\
\hline Dentes restaurados & 65 & 2.6 & 78.31 & 316 & 11.29 & 73.67 & 75 & 3.95 & 18.16 & 456 & 6.33 & 49.29 \\
\hline Dentes Perdidos & 0 & 0 & 0.00 & 93 & 3.32 & 21.68 & 334 & 17.58 & 80.87 & 427 & 5.93 & 46.15 \\
\hline DMFT & 83 & 3.32 & 100.0 & 429 & 15.32 & 100.0 & 413 & 21.74 & 100.00 & 925 & 12.85 & 100.00 \\
\hline
\end{tabular}

Fonte: Autores.

No que diz respeito à autopercepção, houve significância estatística ao cruzar a condição de dentes e gengivas e a necessidade de ir ao dentista, ou seja, aqueles indivíduos que consideravam seus dentes ruins referiram que deveriam procurar atendimento odontológico $(\mathrm{p}=0,024)$. Entretanto, não foi encontrada estatística significativa $(\mathrm{p}=0,802)$ entre a real condição de saúde bucal e a necessidade de procurar atendimento odontológico (Tabela 3).

Tabela 3. Análise comparativa entre as condições dentárias e gengivais bem como a necessidade de ir ao dentista, em pessoas com deficiência visual, participantes de uma instituição no município de São José do Rio Preto, São Paulo. 2019.

\begin{tabular}{|c|c|c|c|c|c|}
\hline \multicolumn{6}{|c|}{ Você acha que precisa ir ao dentista? } \\
\hline Variáveis & \multicolumn{2}{|c|}{ Não } & \multicolumn{2}{|c|}{ Sim } & \multirow[t]{2}{*}{ p-valor } \\
\hline & $\mathrm{n}$ & $\%$ & $\mathrm{n}$ & $\%$ & \\
\hline \multicolumn{6}{|l|}{$\begin{array}{l}\text { Como estão seus dentes e } \\
\text { gengiva? }\end{array}$} \\
\hline Ruins & 06 & 8.30 & 27 & 37.50 & \multirow{2}{*}{$0.024^{*}$} \\
\hline Bons & 18 & 25.00 & 21 & 29.20 & \\
\hline \multicolumn{6}{|l|}{ CPOD } \\
\hline Alto & 12 & 16.67 & 21 & 29.17 & \multirow{3}{*}{$0.802^{*}$} \\
\hline Baixo & 12 & 16.67 & 27 & 37.50 & \\
\hline Total & 24 & 33,33 & 48 & 66,67 & \\
\hline
\end{tabular}

*Teste Qui-quadrado. Fonte: Autores.

A percepção da saúde bucal foi comparada entre os grupos de cegueira total e baixa visão, considerando o impacto causado na qualidade de vida, de acordo com as dimensões avaliadas: limitação funcional, dor física, desconforto psicológico, limitação física, limitação psicológica e limitação social. A dimensão desconforto psicológico foi estatisticamente significativa ( $\mathrm{p}=0,0008)$, ou seja, o grupo com cegueira total apresentou maior impacto na qualidade de vida do que o grupo com baixa visão. Estatísticas significativas foram encontradas em relação ao CPO-D na dimensão limitação funcional ( $\mathrm{p}=0,0071)$ : embora o CPOD tenha sido considerado alto, não afetou a qualidade de vida dos indivíduos (Tabela 4). 
Tabela 4. Impacto da saúde bucal na qualidade de vida dos deficientes visuais, separados por dimensões de acordo com o tipo de deficiência e o nível de CPOD, participantes de uma instituição no município de São José do Rio Preto, São Paulo. 2019.

\begin{tabular}{|c|c|c|c|c|c|c|c|c|c|c|}
\hline \multirow{2}{*}{ Variáveis } & \multicolumn{2}{|c|}{ Cegueira Total } & \multicolumn{2}{|c|}{ Baixa Visão } & \multirow{2}{*}{$p$-valor } & \multicolumn{2}{|c|}{ CPOD Alto } & \multicolumn{2}{|c|}{ CPOD Baixo } & \multirow{2}{*}{$p$-valor } \\
\hline & $\mathrm{n}$ & $\%$ & $\mathrm{n}$ & $\%$ & & $\mathrm{n}$ & $\%$ & $\mathrm{n}$ & $\%$ & \\
\hline \multicolumn{11}{|l|}{ Limitação funcional } \\
\hline Sem impacto & 31 & 43.06 & 35 & 48.61 & \multirow{2}{*}{$0.198^{*}$} & 27 & 37.50 & 39 & 54.17 & \multirow{2}{*}{$0.0071^{*}$} \\
\hline Com impacto & 05 & 6.94 & 01 & 1.39 & & 06 & 8.33 & 0 & 0.00 & \\
\hline \multicolumn{11}{|l|}{ Dor física } \\
\hline Sem impacto & 29 & 40.28 & 33 & 45.83 & \multirow{2}{*}{$0.307^{*}$} & 28 & 38.89 & 34 & 47.22 & \multirow{2}{*}{$1.000 *$} \\
\hline Com impacto & 07 & 9.72 & 03 & 4.17 & & 05 & 6.94 & 05 & 6.94 & \\
\hline \multicolumn{11}{|c|}{ Desconforto psicológico } \\
\hline Sem impacto & 14 & 19.44 & 29 & 40.28 & \multirow{2}{*}{$0.0008^{* *}$} & 26 & 36.11 & 29 & 40.28 & \multirow{2}{*}{$0.871^{* *}$} \\
\hline Com impacto & 22 & 30.56 & 07 & 9.72 & & 07 & 9.72 & 10 & 13.89 & \\
\hline \multicolumn{11}{|l|}{ Limitação física } \\
\hline Sem impacto & 27 & 37.50 & 33 & 45.83 & \multirow{2}{*}{$0.111^{*}$} & 27 & 37.50 & 37 & 51.39 & \multirow{2}{*}{$0.131^{*}$} \\
\hline Com impacto & 09 & 12.50 & 03 & 4.17 & & 06 & 8.33 & 02 & 2.78 & \\
\hline \multicolumn{11}{|l|}{ Limitação psicológica } \\
\hline Sem impacto & 30 & 41.67 & 35 & 48.61 & \multirow{2}{*}{$0.106^{*}$} & 32 & 44.44 & 33 & 45.83 & \multirow{2}{*}{$0.116^{*}$} \\
\hline Com impacto & 06 & 8.33 & 01 & 1.39 & & 01 & 1.39 & 06 & 8.33 & \\
\hline \multicolumn{11}{|l|}{ Limitação social } \\
\hline Sem impacto & 35 & 48.61 & 33 & 45.83 & \multirow{2}{*}{$0.614^{*}$} & 33 & 45.83 & 35 & 48.61 & \multirow{2}{*}{$0.120^{*}$} \\
\hline Com impacto & 01 & 1.39 & 03 & 4.17 & & 0 & 0.00 & 04 & 5.56 & \\
\hline Total & 36 & 50.0 & 36 & 50.0 & & 33 & 45.83 & 39 & 54.17 & \\
\hline
\end{tabular}

* Teste Exato de Fisher. ** Teste Qui-quadrado. Fonte: Autores.

A comparação entre a percepção de saúde bucal e a condição bucal real não foi estatisticamente significativa, ou seja, a percepção de bom ou ruim não está associada a um CPO-D alto ou baixo (Tabela 5).

Tabela 5. Análise comparativa entre a percepção da saúde bucal e o índice CPOD, em pessoas com deficiência visual, participantes de uma instituição no município de São José do Rio Preto, São Paulo. 2019.

\begin{tabular}{|c|c|c|c|c|c|}
\hline \multirow{3}{*}{ Variáveis } & \multicolumn{4}{|c|}{ CPOD } & \multirow{3}{*}{$p$-valor } \\
\hline & \multicolumn{2}{|c|}{ Alto } & \multicolumn{2}{|c|}{ Baixo } & \\
\hline & $\mathrm{n}$ & $\%$ & $\mathrm{n}$ & $\%$ & \\
\hline Percepção de saúde & & & & & \multirow{4}{*}{$0.937 *$} \\
\hline Boa & 18 & 25.0 & 22 & 30.6 & \\
\hline Ruim & 15 & 20.8 & 17 & 23.6 & \\
\hline Total & 33 & 45.8 & 39 & 54.2 & \\
\hline
\end{tabular}

* Teste Qui- quadrado. Fonte: Autores. 


\section{Discussão}

Neste estudo, mediante análise da amostragem total, observou-se a ocorrência de uma ligeira superioridade no número de participantes do gênero masculino, o que se assemelha a outros estudos da área (Singh, Dhawan, Gaurav, Rastogi, \& Singh, 2017; Tagelsir, Khogli, \& Nurelhuda, 2013), razão na qual demonstra que o número de homens com deficiência visual é maior do que o número de mulheres. Um fator que merece destaque em relação a questão é que, ao considerar a cegueira decorrente de doenças sistêmicas não controladas, essa diferença numérica entre os gêneros justifica-se devido aos hábitos de prevenção, que costumam ser mais associados às mulheres do que aos homens (Gomes, Nascimento, \& Araújo, 2007), o que permite a redução do número de casos femininos.

Para avaliação da condição bucal dos deficientes visuais, o índice CPO-D foi analisado separadamente por faixa etária, semelhante a metodologia estabelecida para a Pesquisa Nacional de Saúde Bucal (SB Brasil 2010) (Brasil, 2012). Dessa forma, verificou-se que o CPO-D médio para a faixa etária de 5 a 18 anos foi de 3,3, com predomínio de dentes restaurados, semelhante aos achados de Shetty et. al. (2010), em que o CPO-D médio foi de 4,9, no entanto com predomínio de dentes cariados. Em relação aos achados da SB Brasil, o CPO-D médio foi de 2,1, para a população de 12 anos e 4,2 para a faixa etária de 15 a 19 anos (Brasil, 2012), significando que a condição bucal dos deficientes visuais nessa faixa etária se assemelha à da população em geral.

A faixa etária de 22 a 49 anos também se assemelhou aos resultados obtidos pela SB Brasil (CPO-D médio 16,7) ao apresentar um CPO-D médio de 15,3, composto em sua maioria por dentes restaurados. Para a faixa etária de 50 a 80 anos, o CPO-D médio foi de 21,7, composto principalmente por dentes perdidos, semelhante ao estudo de Souza Filho et. al. (2010) para a população de 45 a 63 anos e inferior ao resultado da SB Brasil (Brasil, 2012) para a população de 65 a 74 anos, que apresentaram CPO-D médio de 20,5 e 27,1, respectivamente, ambos com predomínio de dentes perdidos.

O CPO-D total médio obtido foi de 12,8 para a idade média de 34,5 anos, com prevalência de dentes restaurados, assim como apresentado por Maciel et. al. (2009), que observou um CPO-D médio total de 10,3 para o mesmo tipo de população. No entanto, a média geral encontrada pelo SB Brasil 2010 (Brasil, 2012) foi de 17,5, valor superior à média encontrada para a população estudada.

Porém, apesar de não apresentarem grandes discrepâncias com a população geral do país, os índices CPO-D encontrados ainda são elevados para algumas faixas etárias. Isso se deve à dificuldade de a pessoa com deficiência reconhecer as doenças bucais de forma precoce, ou ainda diante da complexidade em remover o biofilme e utilizar o fio dental de maneira adequada e eficiente. Esses fatores realçam a necessidade de promover ações de saúde e orientações de higiene, específicas para esses indivíduos (Brasil, 2013).

Atualmente, os programas educativos-preventivos comumente utilizados, não possuem eficácia significativa para os portadores de deficiência visual, já que os mesmos dependem muito mais de sensações táteis e auditivas para o aprendizado (Cui, Lin, Lo, Tao, Zhou, \& Zhi, 2017) do que dos meios convencionais de educação e publicidade de prevenção utilizados na esfera da saúde pública. Dessa forma, as técnicas a serem empregadas devem sofrer adequações para respeitar e atender as limitações destes pacientes.

Uma alternativa que pode ser fundamental para o alcance desse público específico e que permitiria uma abordagem mais inclusiva é a adaptação das técnicas ao sistema Braille, uma vez que facilitaria a compreensão dos pacientes e dessa forma possibilitaria que uma parcela significativa passasse a ter mecanismos adequados e eficientes para realização de sua higiene bucal e consequente prevenção de doenças.

Apesar do Sistema Braile ser uma opção viável, como parte da amostra deste estudo comprova, nem todos os indivíduos possuem o domínio do código, o que tornaria o uso exclusivo desta ferramenta algo nem sempre conveniente. Sendo assim, entende-se que para melhor atender as necessidades dos portadores de deficiência visual, é necessário a união de 
diversas técnicas e abordagens para que o ensino seja feito de forma clara, inclusiva e efetiva. Treinar práticas preventivas de controle de placa bacteriana de forma paciente, seja através de escovação manual ou elétrica, com a utilização de dentifrícios fluoretados, podem ser alternativas benéficas para a redução dos problemas bucais (Kumar, Konde, Raj, \& Agarwal, 2012).

Em relação a autopercepção, assim como demonstrado por Souza Filho et al. (2010), observou-se que a maioria dos entrevistados classificou seus dentes e gengivas como sendo bons ou muito bons. É importante ressaltar que na referida categoria, a forma como o indivíduo se auto avalia, pode não estar necessariamente associada à necessidade de ir ao dentista, uma vez que se trata de uma medida subjetiva que proporciona informações essenciais, mas não substitui um exame clínico feito por um profissional habilitado.

Neste estudo, também, observou-se uma significativa porcentagem de pessoas que avaliaram a condição dos dentes e gengivas em situação inapropriada e consideraram a necessidade de visitarem o dentista. Esta evidência corrobora com o estudo de Santos et al. (2015) que, ao avaliar a referida morbidade, constatou que a maioria dos entrevistados relatou a necessidade de tratamento odontológico $(91,4 \%)$.

A percepção sobre a saúde bucal geralmente está associada ao desconforto do indivíduo, ou seja, a pessoa percebe que sua cavidade oral está saudável quando não apresenta dores, mau hálito ou qualquer outro tipo de interferência. No entanto, embora esse indivíduo tenha um alto índice CPOD, desde que não afete suas necessidades básicas, essa condição não interfere em sua noção avaliativa (Singh, Dhawan, Gaurav, Rastogi, \& Singh, 2017). Isso pode ser verificado neste estudo ao analisarmos que a maioria das pessoas com deficiência visual, considera sua saúde bucal boa, apesar de apresentar um índice CPOD fora do padrão e não se enquadrar em parâmetros saudáveis. A condição oral e a percepção sobre a saúde bucal não apresentaram diferenças estatísticas, ou seja, a consciência não está relacionada à condição real, quando se refere a pessoas com deficiência visual.

Já o Perfil de Impacto na Saúde Bucal é avaliado por meio da perspectiva que cada dimensão tem na qualidade de vida da pessoa com deficiência visual. Neste estudo, foi comparado o impacto entre os grupos de cegueira total e baixa visão, de forma que a dimensão do desconforto psicológico foi estatisticamente significativa. Assim, nesta extensão, a repercussão causada na qualidade de vida foi maior no grupo com cegueira total do que no grupo com baixa visão. Isso porque a cegueira total gera ainda mais limitações no cotidiano do indivíduo, o que ocasiona maior desconforto psicológico e insegurança quanto à sua saúde bucal (Teixeira, 2006). O mesmo domínio foi prevalente no estudo de Guerra et al. (2014) ao avaliar a saúde bucal de trabalhadores e como isso os atingia.

Quanto ao CPO-D, contatou-se considerável estatística na dimensão da limitação funcional, o que significa que ter um CPO-D elevado não impactou na qualidade de vida da pessoa cega. Esse resultado é contrário ao que foi verificado no estudo de Garbado et al. (2013) sobre a autopercepção da saúde bucal segundo o Oral Health Impact Profile. Neste estudo, foi encontrada associação positiva entre os piores indicadores e a pior condição de saúde bucal autorreferida. Isso evidencia a influência de fatores micro e macrodimensionais, com repercussões na qualidade de vida das pessoas. Assim, o CPO- D elevado apresenta chances significativamente maiores de influenciar na qualidade de vida.

Embora os resultados apresentados possuam relevância cientifica, algumas limitações podem ser observadas, destacando-se o fato da amostra ser de um único lugar, com limitado número de participantes e grande variação de idadespodendo gerar possível viés- e a ausência de artigos atuais sobre o tema na literatura, principalmente estudos que possuam amostra com características sociodemográficas semelhantes à deste estudo. Novos estudos devem ser considerados no intuito de aprofundar os conhecimentos sobre a temática. 


\section{Conclusão}

Os deficientes visuais possuem uma boa percepção da sua saúde bucal e sua condição bucal é compatível com a média da população brasileira. No entanto, essa média ainda é elevada e indica a necessidade de promover ações de saúde bucal específicas para melhorar a condição desses indivíduos.

\section{Agradecimentos}

O presente trabalho foi realizado com apoio da Coordenação de Aperfeiçoamento de Pessoal de Nível Superior -Brasil (CAPES) -Código de Financiamento 001

\section{Referências}

Al Sarheed, M., Bedi, R., Alkhatib, M. N. \& Hunt, N. P. (2006). Dentists attitude and practices towards provision of orthodontic treatment for children with visual and hearing impairments. Spec Care Dentist, 26 (1), 30-6. 10.1111/j.1754-4505.2006.tb01507.x

Amorim, E. G., Medeiros Neta, O. M. de \& Guimarães, J. (2020). Para uma nova arte de viver: os espaços e as práticas de reabilitação da pessoa com deficiência visual. Research, Society and Development, 9 (8), e310985445. 10.33448/rsd-v9i8.5445.

Ayres, M., Ayres Júnior, M., Ayres, D. L. \& Santos, A. S. dos. (2007). BioEstat 5.0: aplicações estatísticas nas áreas das Ciências Biológicas e Médicas. Belém, PA: Sociedade Civil Mamirauá.

Barbosa, T. S. \& Gavião, M. B. D. (2011). Qualidade de vida e saúde bucal em crianças - parte II: versão brasileira do Child Perceptions Questionnaire. Ciência \& Saúde Coletiva, 16 (7) 3267-3276.

Batista, C. G., Turrini, C. C., Moraes, A. B. A. \& Rolim, G. S. (2003). A odontologia e as pessoas com deficiência visual. Jornal Brasileiro de OdontoPsicologia e Odontologia para Pacientes Especiais, 1 (2), 170-174.

Brasil. (2010). Ministério da Saúde. Secretaria de Atenção à Saúde. Departamento de Ações Programáticas e Estratégicas. Política Nacional de Saúde da Pessoa com Deficiência. Brasília: Ministério da Saúde.

Brasil. (2011). Decreto no 7.612 de 17 de Novembro, 2011. Estabelece o Plano Nacional dos Direitos da Pessoa com Deficiência: Plano Viver Sem Limites. Brasília: Diário Oficial da República Federativa do Brasil.

Brasil. (2013). Viver Sem Limite: Plano Nacional dos Direitos da Pessoa com Deficiência. Brasília: Secretaria de Direitos Humanos

Brasil. (2017). Ministério da Saúde. Portaria de Consolidação nº 2, de 28 de setembro de 2017. Consolidação das normas sobre as políticas nacionais de saúde do Sistema Único de Saúde. Diário Oficial [da] República Federativa do Brasil. Seção Suplemento, 61-192.

Brasil. (2012) Ministério da Saúde. Secretaria de Atenção à Saúde. Secretaria de Vigilância em Saúde. SB Brasil 2010: Pesquisa Nacional de Saúde Bucal: resultados principais. Brasília: Ministério da Saúde.

Brown, D. (2008). An observational study of oral hygiene care for visually impaired children. Tese. Glasgow (UK): University of Glasgow.

Cui, T. Q., Lin, H. C., Lo, E. C. M., Tao, Y., Zhou, Y. \& Zhi, Q. H. (2017). Randomized clinical trial on the efficacy of electric and manual toothbrushes in plaque removal and gingivitis control in visually impaired school students. Quintessence Int, 48 (6), 481-486. 10.3290/j.qi.a38126. PMID: 28439574.

Fundação Dorina Nowill para Cegos. (2021). https://www.fundacaodorina.org.br/a-fundacao/deficiencia-visual/estatisticas-da-deficiencia-visual/>

Gabardo, M. C. L., Moysés, S. T. \& Moysés, S. J. (2013). Autopercepção de saúde bucal conforme o Perfil de Impacto da Saúde Bucal (OHIP) e fatores associados: revisão sistemática. Rev Panam Salud Publica, 33 (6), 439-445.

Gil, N. (2000). Deficiência visual. Secretaria de Educação a Distância Ministério da Saúde. MEC.

Gomes, R., Nascimento, E. F. do. \& Araújo, F. C. de. (2007). Por que os homens buscam menos os serviços de saúde do que as mulheres? As explicações de homens com baixa escolaridade e homens com ensino superior. Cad. Saúd Publ, 3 (3), 565-574. 10.1590/S0102-311X2007000300015.

Guerra, M. J. C., Greco, R. M., Leite, I. C. G., Ferreira, E. F. \& Paula, M. V. Q. (2014). Impact of oral health conditions on the quality of life of workers. Ciênc Saúde Coletiva, 19 (12), 4777-4786. 10.1590/1413-812320141912.21352013.

Honneth, A. (2013). O eu no nós: reconhecimento como força motriz de grupos. Sociologias, 15 (33), 56-80.

Instituto Brasileiro de Geografia e Estatística -IBGE. (2010). Censo Demográfico. biblioteca.ibge.gov.br/index.php/bibliotecacatalogo?view=detalhes\&id=264529

Jokovic, A., Locker, D., Stephens, M., Kenny, D. \& Thompson, B. (2003). Measuring parental perceptions of child oral health-related quality of life. J Public Health Dent, 63 (2), 67-72. 10.1111/j.1752-7325.2003.tb03477.x.

Kumar, S., Konde, S., Raj, S. \& Agarwal, M. (2012). Effect of oral health education and fluoridated dentifrices on the oral health status of visually impaired children. Contemp Clin Dent, 3 (4), 398-401. 10.4103/0976-237X.107425. 
Research, Society and Development, v. 10, n. 8, e47210817499, 2021

(CC BY 4.0) | ISSN 2525-3409 | DOI: http://dx.doi.org/10.33448/rsd-v10i8.17499

Lima, N. C. de, Baptista, T. W. F. \& Vargas, E. P. (2017). Ensaio sobre 'cegueiras': itinerário terapêutico e barreiras de acesso em assistência oftalmológica. Interface - Comunicação, Saúde, Educação, 21 (62), 615-627.

Maciel, M. A. S., Cordeiro, P. M., d’Ávila, S., Godoy, G. P., Alves, R. D. \& Lins, R. D. A. U. (2009). Assessing the oral condition of visually impaired individuals attending the Paraíba Institute of the Blind. Rev Odonto Ciênc, 24 (4),354-360.

Magill, R. A. (2000). Aprendizagem motora: conceitos e aplicações. Edgard Blücher.

Oliveira, A. X. da S., Silva, A. G. da, Dias, E. C. S. C. \& Martins, L. de A. R. (2020). Inclusão Escolar e baixa visão: um Relato de Experiência. Research, Society and Development, 9 (1), e195911769. 10.33448/rsd-v9i1.1769.

Oliveira, B. H. \& Nadanovsky, P. (2005). Psychometric properties of the Brazilian version of the Oral Health Impact Profile-short form. Community Dent Oral Epidemiol, 33 (4), 307-14. 10.1111/j.1600-0528.2005.00225.x

Rath, I. B. S., Bosco, V. L., Almeida, I. C. S. \& Moreira, E. A. M. (2001). Atendimento odontológico para crianças portadoras de deficiência visual. Arquivos em odontologia, 37 (2), 183-8, 2001.

Santos, M. L. M. F., Cruz, S. S., Gomes Filho, I. S., Soares, J. S. P., Figueiredo, A. C. M. G. \& Coelho, C. M. (2015). Satisfação dos usuários adultos com a atenção em saúde bucal na estratégia de saúde da família. Cad Saúde Coletiva, 23 (2), 163-171. 10.1590/1414-462X201500020057.

Shetty, V., Hegde, A. M., Bhandary, S. \& Rai, K. (2010). Oral health status of the visually impaired children: a South Indian. J Clin Pediatr Dent, 34 (3), $213-$ 216. 10.17796/jcpd.34.3.j4781g2w8891848u

Silva, L. de P., Marcelino, J. F. de Q., Gagliardo, H. G. R. G., Santos, K. C. B. M. dos \& Albuquerque, R. C. (2020). Habilidades de interação social e o brincar de crianças com cegueira ou baixa visão em contexto escolar. Research, Society and Development, 9 (9), e135996559. 10.33448/rsd-v9i9.6559.

Singh, A., Dhawan, P., Gaurav, V., Rastogi, P. \& Singh, S. (2017). Assessment of oral health-related quality of life in 9-15 year old children with visual impairment in Uttarakhand, India. Dent Res J, 14 (1), 43-49.

Slade, G. D. (1997). Derivation and validation of a short-form oral health impact profile. Community Dent Oral Epidemiol, 25 (4), 284-290. 10.1111/j.16000528.1997.tb00941.x

Souza Filho, M. D., Nogueira, S. D. M. \& Martins, M. C. C. (2010). Avaliação da saúde bucal de deficientes visuais em Teresina-PI. Arq Odontol, 45 (2), 6674.

Tagelsir, A., Khogli, A. E. \& Nurelhuda, N. M. (2013). Oral health of visually impaired schoolchildren in Khartoum State, Sudan. BMC Oral Health, 13, 33. $10.1186 / 1472-6831-13-33$

Teixeira, L. A. (2006). Controle motor. Manole.

World Health Organization. (2013). Oral health surveys: basic methods. (5th ed.), WHO.

Yalcinkaya, S. E. \& Atalay, T. (2006). Improvement of oral hygiene knowledge in a group of visually impaired students. Oral Health Prev Dent, 4 (4), $243-$ 253. 